\title{
Biosynthesis of Heterocyclic Antibiotics in Actinomycetes and an Approach to Synthesize the Natural Compounds
}

\author{
Hiroyasu Onaka \\ Department of Biotechnology, Toyama Prefectural University
}

(Received Oct. 27, 2006 / Accepted Oct. 27, 2006)

\begin{abstract}
Staurosporine and rebeccamycin are natural antitumor compounds produced by actinomycetes. Indolocarbazole biosynthetic gene clusters have been cloned from both Streptomyces sp. TP-A0274 and Lechevalieria aerocolonigenes ATCC39243. Staurosporine biosynthetic gene cluster consists of 15 orfs spanning $22 \mathrm{~kb}$, and rebeccamycin biosynthetic gene cluster consists of 10 orfs spanning $16 \mathrm{~kb}$. Gene disruption and bioconversion experiments revealed that indole-3-pyruvic acid, which is derived from tryptophan, is coupled to yield chromopyrrolic acid, and then this dicarboxylic bisindole compound is transformed into the indolocarbazole skeleton via two oxidation steps. Goadsporin is another heterocyclic antibiotic produced by Streptomyces sp. TP-A0584. It is a polypeptide antibiotic containing the thiazole and oxazole rings. The biosynthetic goadsporin gene cluster consists of 10 genes: a structural gene $\operatorname{god} A$ and nine genes involved in post-translational modification, immunity, and transcriptional regulation. It is particularly noteworthy that godI, which shows high similarity to the subunit of the "signal recognition particle," plays an important role in goadsporin immunity. Furthermore, combinatorial biosynthesis was applied to produce the indolocarbazole and goadsporin analogs, and as a result, 14 analogs of indolocarbazole and 4 of goadsporin were produced.
\end{abstract}

\section{INTRODUCTION}

Staurosporine and rebeccamycin that belong to the same indolocarbazole group of antibiotics are expected to be the main lead compounds of antitumor drugs (Fig. 1) because they are inhibitors of protein kinases ${ }^{1)}$ or DNA topoisomera$\mathrm{ses}^{2)}$. Most indolocarbazole compounds are isolated from actinomycetes: rebeccamycin from Lechevalieria aerocolonigenes ATCC 39243, staurosporine from Streptomyces sp. TP-A0274 and L. aerocolonigenes subsp. staurosporeus AM-2282, K252a from Nocardiopsis sp. K2524), and BE13793C from Streptoverticillium mobaraense BA137935). Although the chemical structures of the indolocarbazole compounds have been noted for many years, little is known about the molecular machinery required for the formation of the indolocarbazole skeleton. Biosynthetic studies conducted with isotope-labeled precursors indicated that the indolocarbazole structure is derived from two molecules of tryptophan, probably intermediated via indolepyruvic $\operatorname{acid}^{6-8)}$. On the other hand, cloning of the indolocarbazole biosynthetic genes was not performed until recently because the clue to gene cloning had not been revealed; therefore, the biosynthetic pathway of indolocarbazole compounds was also unknown. Ohuchi et al. reported the cloning of the $n g t$ gene encoding $\mathrm{N}$-glycosyl transferase from $L$. aerocolonigenes ATCC $39243^{9)}$, which is a rebeccamycin producer. They heterologously expressed $n g t$ in Streptomyces lividans and revealed that $n g t$ is responsible for $\mathrm{N}$-glycosylation of the indolocarbazole chromophore, that is, $n g t$ belongs to the rebeccamycin biosynthetic genes. This result triggered the

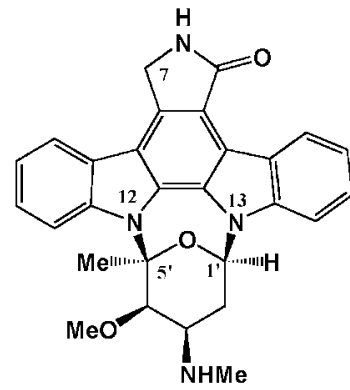

1 Staurosporine

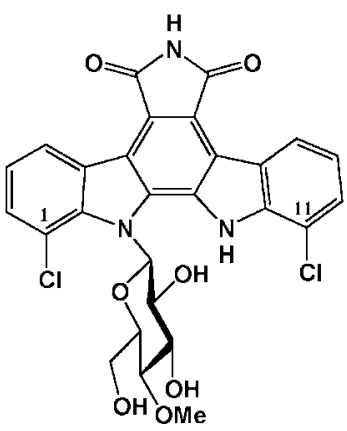

2 Rebeccamycin
Fig. 1. Chemical structures of staurosporine and rebeccamycin.

cloning of the indolocarbazole biosynthetic gene cluster, and the functions proposed for the reb and sta genes have been supported by our independent experimental evidences ${ }^{10,11)}$ and those of other laboratories ${ }^{12,13,14)}$.

Goadsporin, another heterocyclic antibiotic, also has a unique structure containing six heterocyclic rings in the linear backbone (Fig. 1). It is a 19-amino acid long linear polypeptide containing four oxazole and two thiazole rings derived from serine, threonine, or cysteine. We detected this compound in 2000 as a secondary metabolite produced by Streptomyces sp. TP-A0584 ${ }^{15,16)}$. Actinomycetes are well known for their production of a wide variety of polypeptide antibiotics. In particular, numerous nonribosomal peptides have been isolated from actinomycetes; however, the goadsporin-related structures have not yet been reported. 


\section{Indolocarbazole biosynthesis ${ }^{10,11)}$}

(i) Cloning of staurosporine and rebeccamycin biosynthetic genes

To clone the entire set of rebeccamycin biosynthetic genes, a genomic library (100,000 clones) of L. aerocolonigenes was prepared by subcloning Sau3AI-partially digested and dephosphorylated chromosomal DNA into the BamHI site of pKU402. The clones containing the rebeccamycin biosynthetic genes were screened by colony hybridization with a probe designed for $n g t$. A positive cosmid clone designated pREBCN5 was selected and used for further characterization. DNA sequence determination of pREBCN5 revealed that the gene cluster consists of 10 genes spanning $17 \mathrm{~kb}$ as shown in Fig. 2B.

To clone the staurosporine biosynthetic gene cluster, we constructed a genomic cosmid library from Streptomyces sp. TP-A0274, which is a staurosporine producer, and performed colony hybridization with a probe designed for $r e b D$. The selected clone was designated pTYMCsta and used for further characterization. pTYMCsta was constructed with pTOYAMAcos ${ }^{17)}$, which is a bifunctional cosmid vector between actinomycetes and Escherichia coli. The staurosporine biosynthetic gene cluster consists of 15 orfs spanning $20 \mathrm{~kb}$ as shown in Fig. 2A.

A

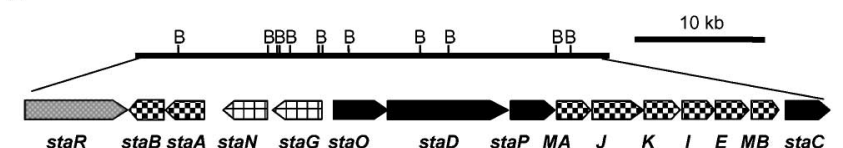

B

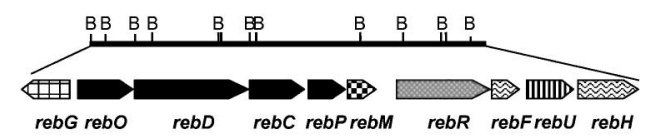

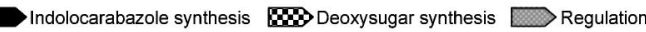
Chlorination ImIm Resistance $\mathrm{Glycosylation}$

Fig. 2. Restriction and organization chromosomal map of the sta locus (A) from Streptomyces TP-A0274 and reb locus (B) from Lechevalieria aerocolonigenes ATCC34294. "B" indicates the Bam HI sites.
The cloned staurosporine biosynthetic gene cluster showed striking similarity to the cloned rebeccamycin gene cluster. Tables 1 and 2 summarize the proposed functions of both the biosynthetic genes.

(ii) Heterologous production of indolocarbazole compounds in S. lividans ${ }^{11,17)}$

The entire sets of both rebeccamycin and staurosporine biosynthetic gene clusters were heterologously expressed in $S$. lividans by using pTOYAMAcos, and the production of these indolocarbazole compounds in the surrogate host was observed. Their productivity in the heterologous hosts was less than that in the original hosts. The amounts of staurosporine (9 days) and rebeccamycin (11 days) produced in the heterologous hosts were 2.6 and $12.1 \mathrm{mg} /$ liter, respectively, whereas those in the original hosts were 10.5 and $132.2 \mathrm{mg} /$ liter, respectively.

(iii) Identification of indolocarbazole biosynthetic pathway $^{10,11,18)}$

We characterized the biosynthetic gene clusters for rebeccamycin and staurosporine through gene disruption experiments by using a newly developed host-vector system for the genus Lechevalieria. The structures of the accumulated products of each disrupted mutant were identified, and the overall biosynthetic pathways of rebeccamycin and staurosporine were proposed. A total of 18 gene disrupted mutants were constructed and identified as the accumulated products. The HPLC profiles of the products are shown in Fig. 3. The accumulated products of the disrupted mutants of $r e b G, r e b P, r e b C, r e b C+r e b G, r e b M$, rebH, staN, staP, staMA, and $s t a M B$ were purified by column chromatography and their chemical structures were determined. Based on these accumulated products and the gene function predicted by the amino acid sequence database searches, we proposed the rebeccamycin biosynthetic pathway as the first indolocarbazole biosynthetic pathway ${ }^{10)}$ (Fig. 4). The indolocarbazole skeleton was biosynthesized from two tryptophan molecules. The gene disruption experiments of both the biosynthetic pathways revealed that indole-3-pyruvic acid, which is derived from tryptophan, is coupled to yield chro-

Table 1. Deduced genes and their proposed functions in the reb cluster.

\begin{tabular}{|c|c|c|c|c|c|}
\hline Gene & $\begin{array}{c}\text { No. of } \\
\text { amino acids }\end{array}$ & Homologous gene & $\begin{array}{l}\% \text { Identity } \\
\text { of product }\end{array}$ & Origin & $\begin{array}{c}\text { Accession } \\
\text { no. }\end{array}$ \\
\hline$r e b G$ & 421 & staG: N-glycosyltransferase & 59.3 & Streptomyces sp. TP-A0274 (staurosporine) & AB088119 \\
\hline $\mathrm{rebO}$ & 473 & $s t a O:$ L-amino acid oxidase & 57.0 & Streptomyces sp. TP-A0274 (staurosporine) & AB088119 \\
\hline$r e b D$ & 557 & staD: chromopyrrolic acid synthase & 55.7 & Streptomyces sp. TP-A0274 (staurosporine) & AB088119 \\
\hline$r e b C$ & 529 & staC: monooxygenase & 64.8 & Streptomyces sp. TP-A0274 (staurosporine) & AB088119 \\
\hline rebP & 397 & staP: chytochrome $\mathrm{P} 450$ & 52.7 & Streptomyces sp. TP-A0274 (staurosporine) & AB088119 \\
\hline rebM & 273 & C-27 O-methyltransferase & 50.2 & Amy. mediterranei (rifamycin) & AF040570 \\
\hline rebR & 958 & staR: luxR type transcriptional regulator & 35.0 & Streptomyces sp. TP-A0274 (staurosporine) & AB088119 \\
\hline rebF & 170 & flavin reductase & 50.3 & S. carzinostaticus (neocarzilin) & AB097904 \\
\hline$r e b U$ & 426 & aviJ: $\mathrm{Na}^{+} / \mathrm{H}^{+}$antiporter & 37.4 & S. viridochromogenes (avilamycin) & AF333038 \\
\hline $\mathrm{rebH}$ & 530 & prnA: tryptophan halogenase & 55.7 & P. chlororaphis (pyrrolnitrin) & AF161182 \\
\hline$r e b T$ & 473 & $m c t$ : mitomycin $\mathrm{C}$ translocase & 45.6 & S. lavendulae (mitomycin) & AF127374 \\
\hline
\end{tabular}


Table 2. Deduced genes and their proposed functions in the sta cluster.

\begin{tabular}{|c|c|c|c|c|c|}
\hline Gene & $\begin{array}{c}\text { No. of } \\
\text { amino acids }\end{array}$ & Homologous gene & $\begin{array}{l}\% \text { Identity } \\
\text { of product }\end{array}$ & Origin & $\begin{array}{c}\text { Accession } \\
\text { no. }\end{array}$ \\
\hline staR & 943 & rebR: luxR type transcriptional regulator & 35 & Lec. aerocolonigenes (rebeccamycin) & AB071405 \\
\hline$s t a B$ & 334 & aviE1: dTDP-glucose-4,6-dehydratase & 70 & S. viridochromogenes (avilamycin) & AF333038 \\
\hline staA & 350 & strD: glucose-1-phosphate thymidyltransferase & 69 & S. griseus (streptomycin) & AJ862840 \\
\hline staN & 394 & $\operatorname{dox} A$ : chytochrome $\mathrm{P} 450$ & 27 & S. peucetius (daunorubicin) & U77891 \\
\hline $\operatorname{staG}$ & 446 & rebG: N-glycosyltransferase & 56 & Lec. aerocolonigenes (rebeccamycin) & AB071405 \\
\hline staO & 504 & rebO: L-amino acid oxidase & 53 & Lec. aerocolonigenes (rebeccamycin) & AB071405 \\
\hline staD & 1096 & rebD: chromopyrrolic acid synthase & 55 & Lec. aerocolonigenes (rebeccamycin) & AB071405 \\
\hline staP & 417 & rebP: chytochrome $\mathrm{P} 450$ & 52 & Lec. aerocolonigenes (rebeccamycin) & AB071405 \\
\hline staMA & 276 & rebM: O-methyltransferase & 36 & Lec. aerocolonigenes (rebeccamycin) & AB071405 \\
\hline staJ & 472 & $u r d S:$ 2,3-dehydratase & 68 & S. fradiae (urdamycin) & AF269227 \\
\hline staK & 328 & jadV: 4-ketoreductase & 47 & S. venezuelae (jadomycin B) & AY026363 \\
\hline staI & 369 & orf10: aminotransferase & 81 & Amy. mediterranei (balhimycin) & Y16952 \\
\hline staE & 206 & orf11: 3, 5-epimerase & 71 & Amy. mediterranei (balhimycin) & Y16952 \\
\hline staMB & 280 & mitM: O-methyltransferase & 53 & S. lavendulae (mitomycin) & AF127374 \\
\hline staC & 545 & $r e b C:$ monooxygenase & 64 & Lec. aerocolonigenes (rebeccamycin) & AB071405 \\
\hline
\end{tabular}

A

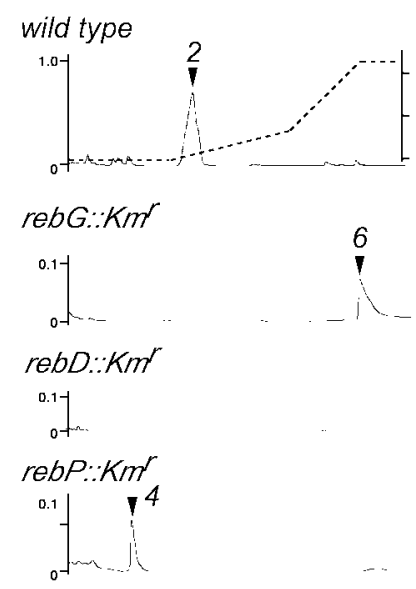

$\triangle r e b C$
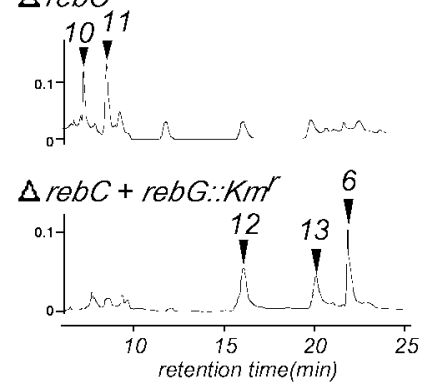

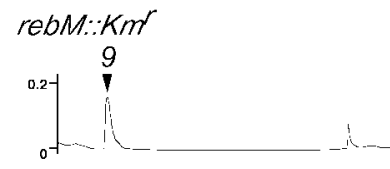

rebH::Km

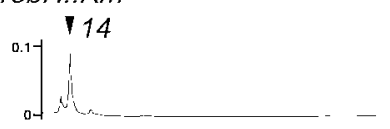

$\triangle r e b R$

0.1.
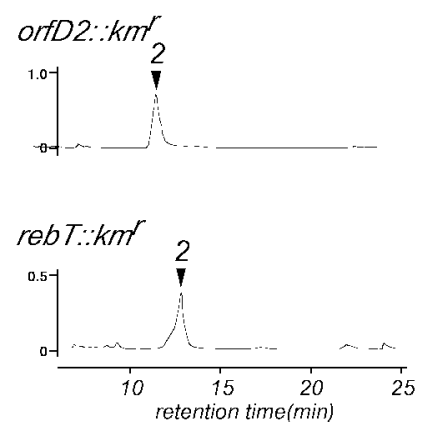

B
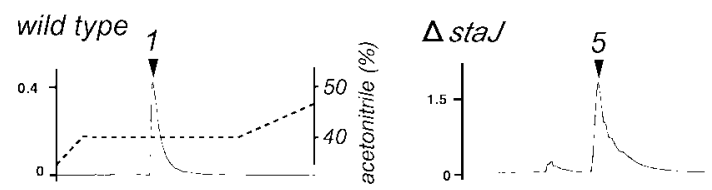

$\Delta \operatorname{stan}$

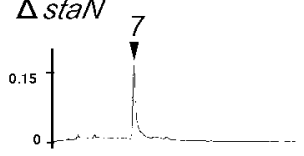

$\Delta$ stal

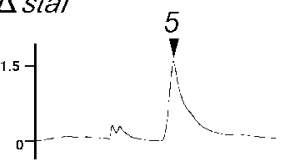

$\Delta s t a D$

0.5

$\Delta$ staE
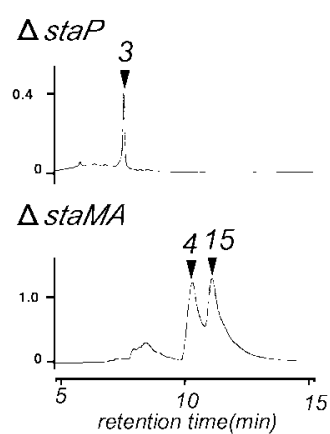

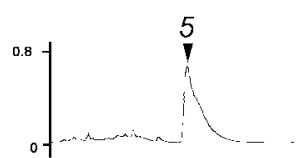

$\triangle \operatorname{staMB}$

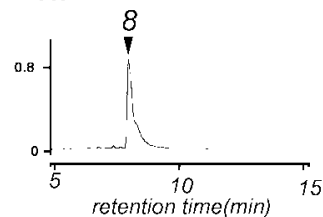

Fig. 3. HPLC chromatograph of the products of the gene-disrupted mutants. HPLC conditions and sample preparation have been described previously ${ }^{10}$. Elution was performed using a linear gradient as indicated on the right-hand scale. A number at each peaks of the HPLC profile indicates the structure number corresponding to Fig. 4 and Fig. 6.

mopyrrolic acid, and then this dicarboxylic bisindole compound is transformed into the indolocarbazole skeleton via the following two oxidation steps (Fig. 4A).

In the rebeccamycin biosynthetic pathway, chlorination of tryptophan by the halogenase RebH is the initial step, and the produced 7-chloro-tryptophan is used as a precursor for indolocarbazole skeleton biosynthesis as described above. The respective indolocarbazole aglycons are added to the distinctive deoxysugars to produce staurosporine or rebeccamycin as shown in Fig. 4B \& C. A major structural difference between staurosporine and rebeccamycin is with regard to the presence of the $\mathrm{C}-\mathrm{N}$ bond between the nitrogen 
A

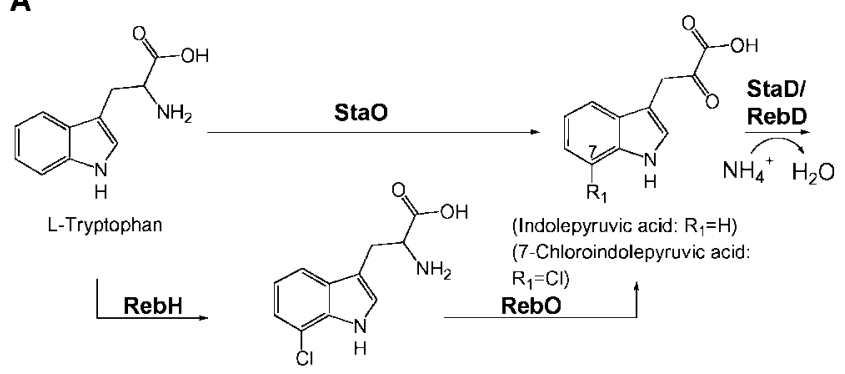

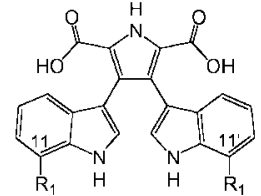

(3 Chromopyrrolic acid: $\mathrm{R}_{1}=\mathrm{H}$ ) (4 11,11'-Dichlorochromopyrrolic acid: $\mathrm{R}_{1}=\mathrm{Cl}$ )

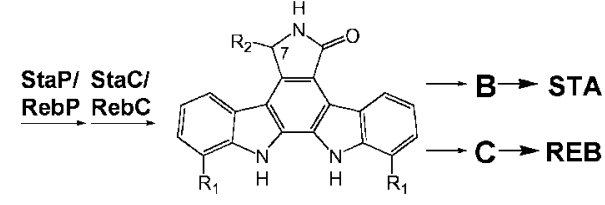

(5 Staurosporine aglycon: $R_{1}$,

$\mathrm{R}_{2}=\mathrm{H}$ )

6 Rebeccamycin aglycon: $\mathrm{R}_{1}=\mathrm{Cl}$.

$\mathrm{R}_{2}=\mathrm{O}$ )

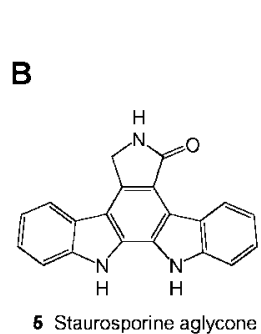

6 Staurosp
(K252c)
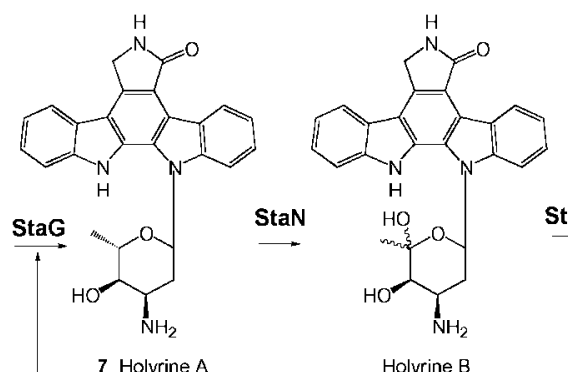

Holyrine B
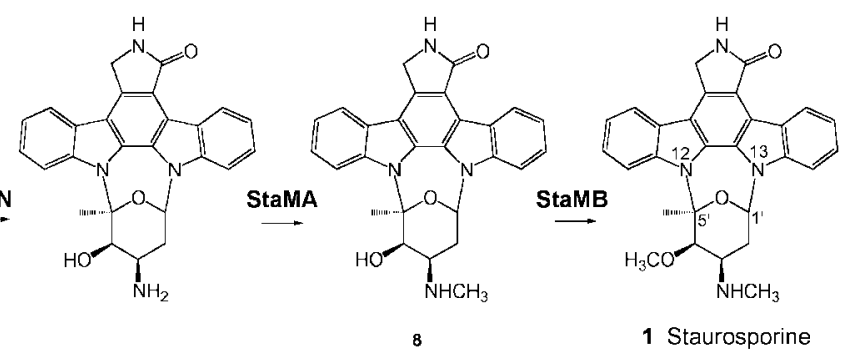

1 Staurosporine

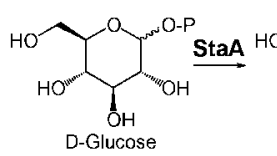<smiles>OC[C@H]1O[C@H](O[GaH2])[C@@H](O)[C@H](O)[C@H]1O</smiles><smiles>CCO[C@H]1OC(C)[C@@H](OCC)C(=O)[C@H]1O</smiles>

$\stackrel{\text { StaE }}{\longrightarrow}$<smiles>C[C@H]1O[C@H](O[GaH2])[C@@H](O)[C@H](O)C1=O</smiles><smiles>CCO[C@H]1O[C@H](C)[C@@H](O)[C@H](O)[C@H]1O</smiles><smiles>C[C@@H]1O[C@H](O[GaH2])CC(=O)[C@H]1O[GaH]</smiles>

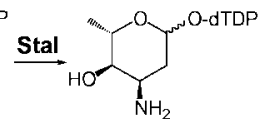

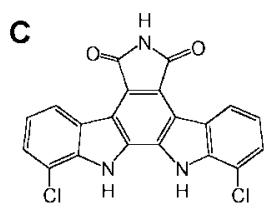

6 Rebeccamycin aglycone

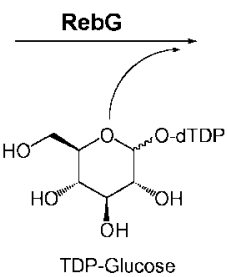

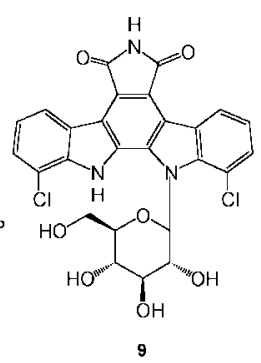

RebM

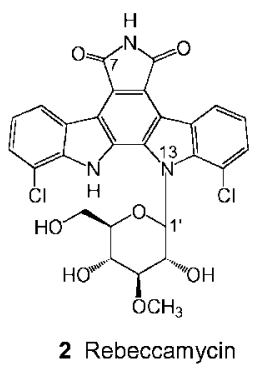

Fig. 4. The proposed overall biosynthetic pathway of the indolocarbazole skeleton (A), staurosporine (B), and rebeccamycin (C).

at N-12 of aglycon and the carbon at C-5' of deoxysugar (Fig. 1). Both these structures possess a glycosidic bond between $\mathrm{N}-13$ and $\mathrm{C}-1$ ' position, and these linkages are catalyzed by $\mathrm{N}$-glycosyltransferases, which are encoded by $s t a G$ or $r e b G$. In the staurosporine structure, there exists an additional unusual C-N bond between N-12 and C-5' position. Through gene disruption and bioconversion experiments, we found that staN, a P450 homolog, is responsible for this unusual C$\mathrm{N}$ bond formation. This was the first example of the $\mathrm{P} 450$ homolog involvement in glycosidic bond formation ${ }^{18)}$. The other major structural difference was with respect to the oxidation state at the $\mathrm{C}-7$ position of the pyrrole ring in the indolocarbazole chromophore. The rebC-disrupted mutant accumulated dichlorochromopyrrolic acid, and the rebPdisrupted mutant accumulated three indolocarbazole chromophores, which differ in their oxidation states at the C-7 position. Bioconversion experiments with 7-deoxo-7-hydroxyrebeccamycin aglycon (12) and 7-deoxorebeccamycin aglycon (13), as an added sample, were conducted to confirm which of these was the real intermediate, but neither compound was converted to rebeccamycin; therefore, we concluded that 12 and $\mathbf{1 3}$ are the shunt products of rebeccamycin biosynthesis. These results suggested that RebP participated in the conversion of CCA to rebeccamycin aglycon, and that RebC controlled the oxidation state at the C-7 site. Very recently, an in vitro study on $\mathrm{StaP}, \mathrm{StaC}$, and $\mathrm{RebC}$ has been reported ${ }^{19)}$. These results indicate that $\mathrm{StaP}$ could produce dichloro derivatives of $\mathbf{6 , 1 2}$, and $\mathbf{1 3}$ directly, and the overall oxidation route is controlled by $\mathrm{StaC}$ and $\mathrm{RebC}$. These results were consistent with those of our gene disruption experiments. 
(iv) Characterization of $\mathrm{StaD}$, the chromopyrrolic acid synthase $^{20)}$

The formation of chromopyrrolic acid, which is the key intermediate of indolocarbazole biosynthesis, is catalyzed by $\mathrm{StaD}$ in staurosporine biosynthesis. We functionally expressed the StaD protein in E. coli and revealed that it is a tetrameric hemoprotein, and that it catalyzes the coupling reaction between two molecules of indole-3-pyruvic acid and $\mathrm{NH}_{4}^{+}$to yield chromopyrrolic acid (Fig. 5).

To date, only two homologs of StaD have been reported; one is RebD (55\% amino acid identity), which is involved in rebeccamycin biosynthesis, and the other is VioB (37\% identity), which is involved in violacein biosynthesis. Recently, the enzymatic mechanism of RebD was studied independently, and the enzymatic reaction was found to be the same as that of $\mathrm{StaD}^{21,22)}$. All proteins belonging to the $\mathrm{StaD}$ family consist of approximately 1000 amino acids, and the deduced amino acid sequences have no significantly conserved domains. It is suggested that the StaD family is a new type of hemoprotein that has a novel structure and function.
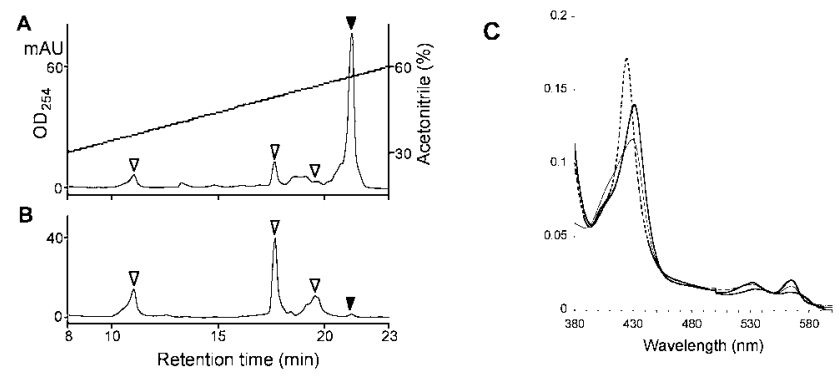

Fig. 5. In vitro characterization of StaD. A and B: HPLC analysis of the StaD enzyme reaction. The HPLC profile (A) when the StaD enzyme is present, and (B) when it is absent. Chromopyrrolic acid is indicated by filled arrowheads, and indole-3-pyruvic acid is indicated by the three nonfilled arrowheads. HPLC and reaction conditions have been described previously ${ }^{20)}$. C: Electronic absorption spectra of nontreated (solid line), $\mathrm{Na}_{2} \mathrm{~S}_{2} \mathrm{O}_{4}$-treated (thick line), and CO-treated (dotted line) forms of $\mathrm{StaD}$, suggesting that $\mathrm{StaD}$ contains heme.

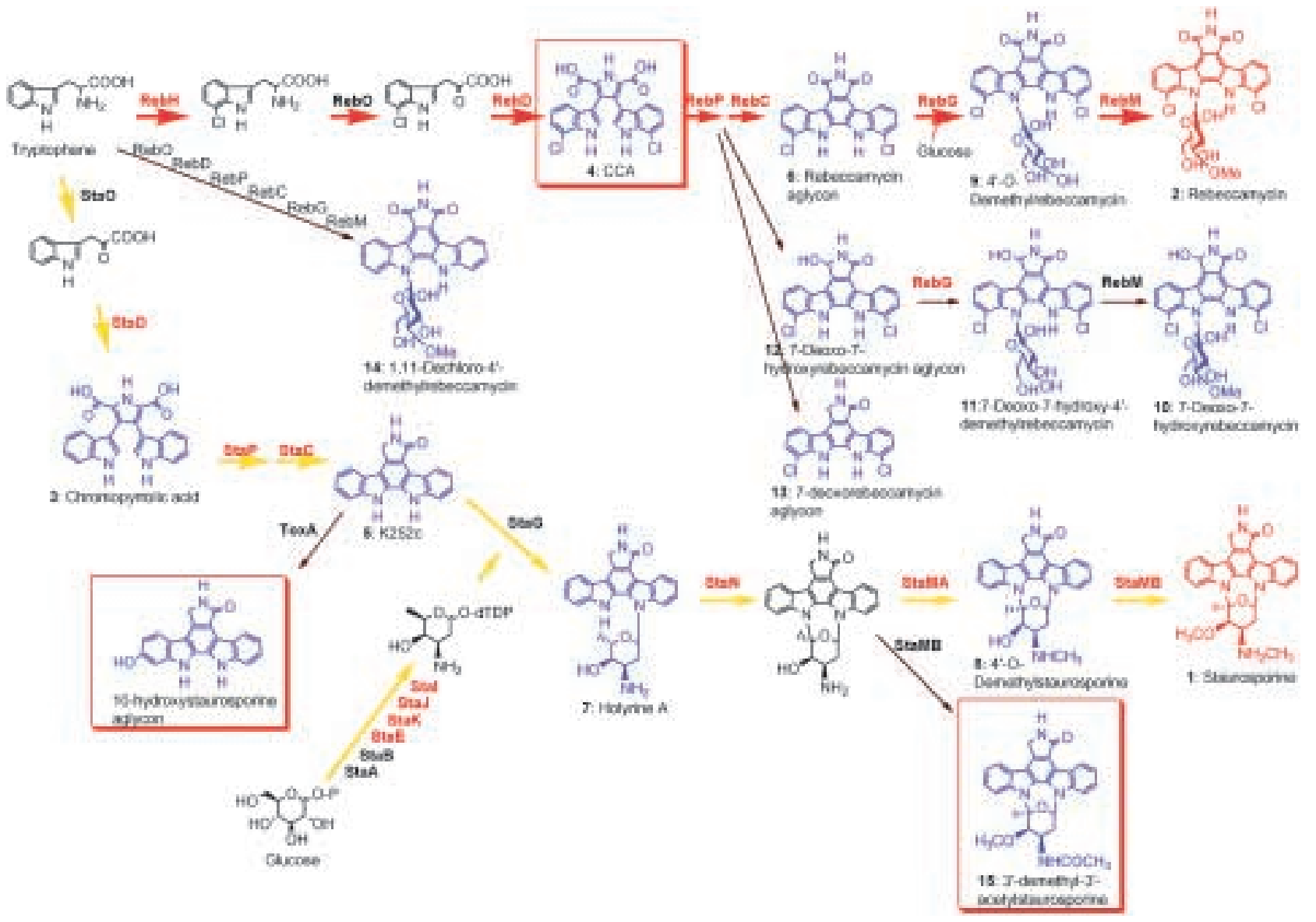

Fig. 6. Rational biosynthesis of the indolocarbazole-related compounds. The red genes are the constructed disrupted mutants. The blue compounds are accumulated in the genetically manipulated strains. The red boxes enclose the novel chemical structures revealed in this study. The red arrowed route indicates the rebeccamycin biosynthetic pathway, and the yellow route indicates the staurosporine biosynthetic pathway. The shunt pathway is indicated by brown arrowed routes. 
(v) Approach to synthesize the natural indolocarbazole compounds ${ }^{23}$

To date, we constructed 18 biosynthetic gene disruptants by using both the indolocarbazole biosynthetic pathways, and 14 types of analogs were produced. Of these recombinant analogs, three were compounds with new structures (Fig. 6). Thus, we have demonstrated that the biosynthetic disruptants provide a basis for the construction of the indolocarbazole analog library.

\section{Goadsporin biosynthesis}

(i) The discovery of goadsporin

Production of secondary metabolites of actinomycetes is influenced by their growth conditions such as temperature, aeration, and medium composition. We developed a new screening method to detect the chemical inducer of secondary metabolite production. The inducing activity was determined by red pigment production in S. lividans. This species is known to possess a complete set of biosynthetic genes for actinorhodin, a red pigment, although it does not produce the red pigment under conventional growth conditions. In this screening test, goadsporin was revealed as a substance that promotes the formation of diffusible red pigment, aerial hyphae, and sporulation in S. lividans under conventional growth condition15).

Goadsporin, a secondary metabolite of Streptomyces sp. TP-A0584, is a 19-amino acid long polypeptide containing four thiazole and two oxazole rings ${ }^{16)}$ (Fig. 10). Goadsporin promotes the secondary metabolism and morphogenesis at low concentrations and induces growth inhibition at high concentrations in actinomycetes. In S. lividans, it promotes the formation of red pigment and sporulation at a concentration of $1 \mu \mathrm{M}$ and inhibits growth at $>1 \mu \mathrm{M}$ (Fig. 7). This activity, which is observed in a wide variety of actinomycetes, is not observed in other organisms. Among 42 tested actinomycetes strains, 36 showed an induction of sporulation and/or secondary metabolite production at low goadsporin concentrations (Fig. 7).

(ii) Cloning of goadsporin biosynthetic gene cluster ${ }^{24)}$

It was presumed that goadsporin is ribosomally biosynthesized, and the precursor sequence is ATVSTILCSGGTLSSAGCV; therefore, the goadsporin biosynthetic gene cluster was cloned from the TP-A0584 genomic library by using a synthetic oligonucleotide probe, which is designed from the amino acid sequence of the goadsporin precursor. Two positive clones were obtained and designated pGSBC1 and pGSBC2. The nucleotide sequencing of the pGSBC1 fragment revealed that the goadsporin ( $g o d)$ gene cluster consisted of 10 orfs. From sequence analysis and database comparison, we proposed the functions of the god gene (Table 3). The proposed biosynthetic pathway for goadsporin is shown in Fig. 8. The godA gene encodes a precursor peptide of goadsporin. The 49-amino acid long godA polypeptide is ribosomally produced, and it is processed by putative goadsporin synthetases, namely, GodD, GodE, GodF, and GodG. These enzymes transform the amino acids at positions $2,5,8,12,15$, and 18 from the $\mathrm{N}$-terminus into heterocycles and the serine residues at positions 4 and 14 into

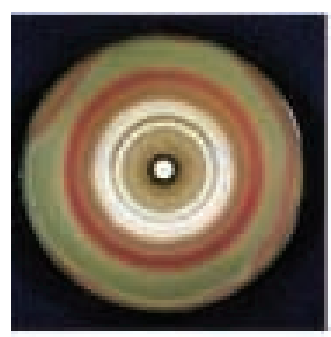

S. lividans TK23

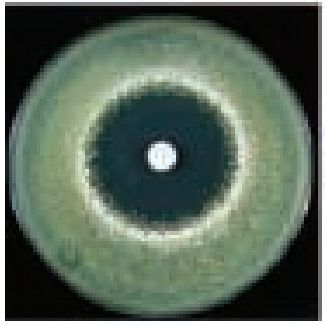

S. hygroscopicus MCRL0401

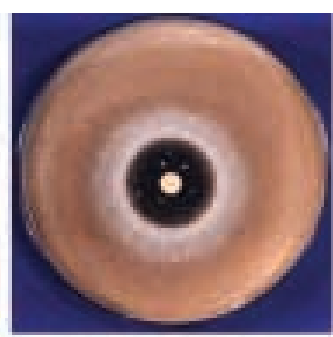

$S$. avermitilis IFO 14893

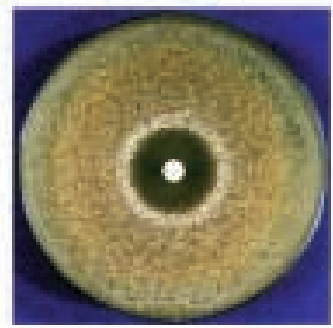

S. spinicoumarensis ATCC29813

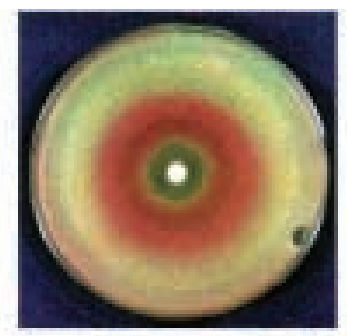

S. coelicolor A3(2)

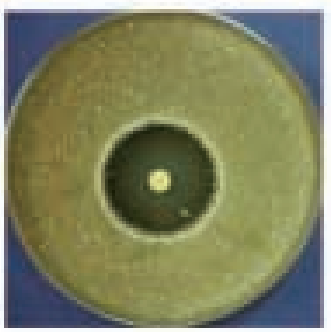

Streptomyces $s p$, TP-A0274

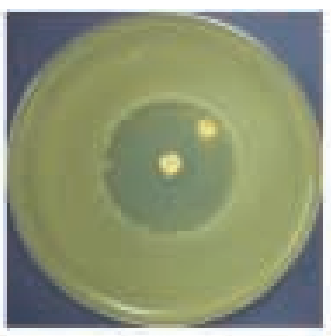

$S$ griseus IFO13350

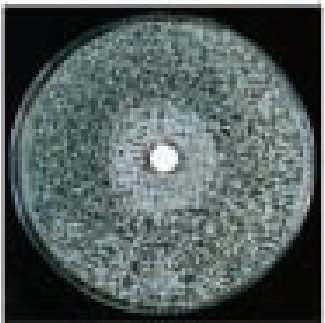

S. griseolus NRRL 3739

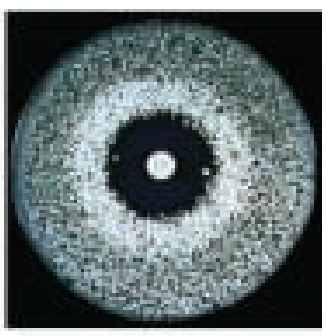

Microbispora sp.

TP-A0184

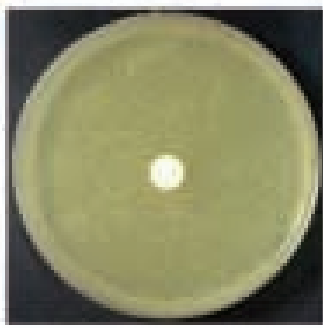

Streptomyces sp. TP-A0584

Fig. 7. Biological activities of goadsporin with regard to sporulation and pigment production in various Streptomyces strains assessed on a solid medium by using $6 \mathrm{nmol}\left(\mathrm{ca} .10 \mu \mathrm{g}\right.$ ) of goadsporin absorbed on paper discs. The assay conditions have been described previously ${ }^{15}$. 
Table 3. Deduced genes and their proposed functions in the god cluster.

\begin{tabular}{|c|c|c|c|c|c|}
\hline Gene & $\begin{array}{c}\text { No. of } \\
\text { amino acids }\end{array}$ & Homologous gene & $\begin{array}{l}\% \text { Identity } \\
\text { of product }\end{array}$ & Origin & $\begin{array}{c}\text { Accession } \\
\text { no. }\end{array}$ \\
\hline $\operatorname{godA}$ & 49 & goadsporin structure gene & & & \\
\hline $\operatorname{godB}$ & 550 & $l k t B$ :Leukotoxin secretion ATP-binding protein & 22.7 & Act. actinomycetemcomitans & X53955 \\
\hline $\operatorname{god} C$ & 557 & $l k t B$ & 18.7 & Act. actinomycetemcomitans & X53955 \\
\hline $\operatorname{godD}$ & 735 & gra-orf12 involved in granaticin biosynthesis & 44.4 & Streptomyces violaceoruber & AJ011500 \\
\hline godE & 522 & $m c b C$ involved in microcinB17 biosynthesis & $25.0 *$ & Escherichia coli & M24253 \\
\hline $\operatorname{godF}$ & 867 & function unknown & & & \\
\hline $\operatorname{god} G$ & 229 & function unknown & & & \\
\hline $\operatorname{godH}$ & 222 & acetyltransferase (putative) & 40.9 & Mycobacterium tuberculosis & NP_215317 \\
\hline $\operatorname{godR}$ & 238 & $\operatorname{brp} A$ : reglator protein of bialaphos biosynthesis & 22.7 & Streptomyces hygroscopicus & M64783 \\
\hline orf1 & 160 & transposase (putative) & 93.8 & Streptomyces avermitilis & NP_828729 \\
\hline orf 2 & 147 & transposase (putative) & 89.8 & S. avermitilis & NP_828730 \\
\hline orf3 & 304 & transposase (putative) & 76.3 & Streptomyces coelicolor A3(2) & NP_631141 \\
\hline $\operatorname{godI}$ & 518 & $f f h$ : signal recognition particle protein & 81.6 & Streptomyces lividans TK21 & AF071565 \\
\hline orf5 & 73 & transposase (putative) & $43.8 *$ & S. avermitilis & NP_821430 \\
\hline orf6 & 128 & transposase (putative) & $42.5^{*}$ & S. avermitilis & NP_821430 \\
\hline
\end{tabular}

*: partial sequence homology

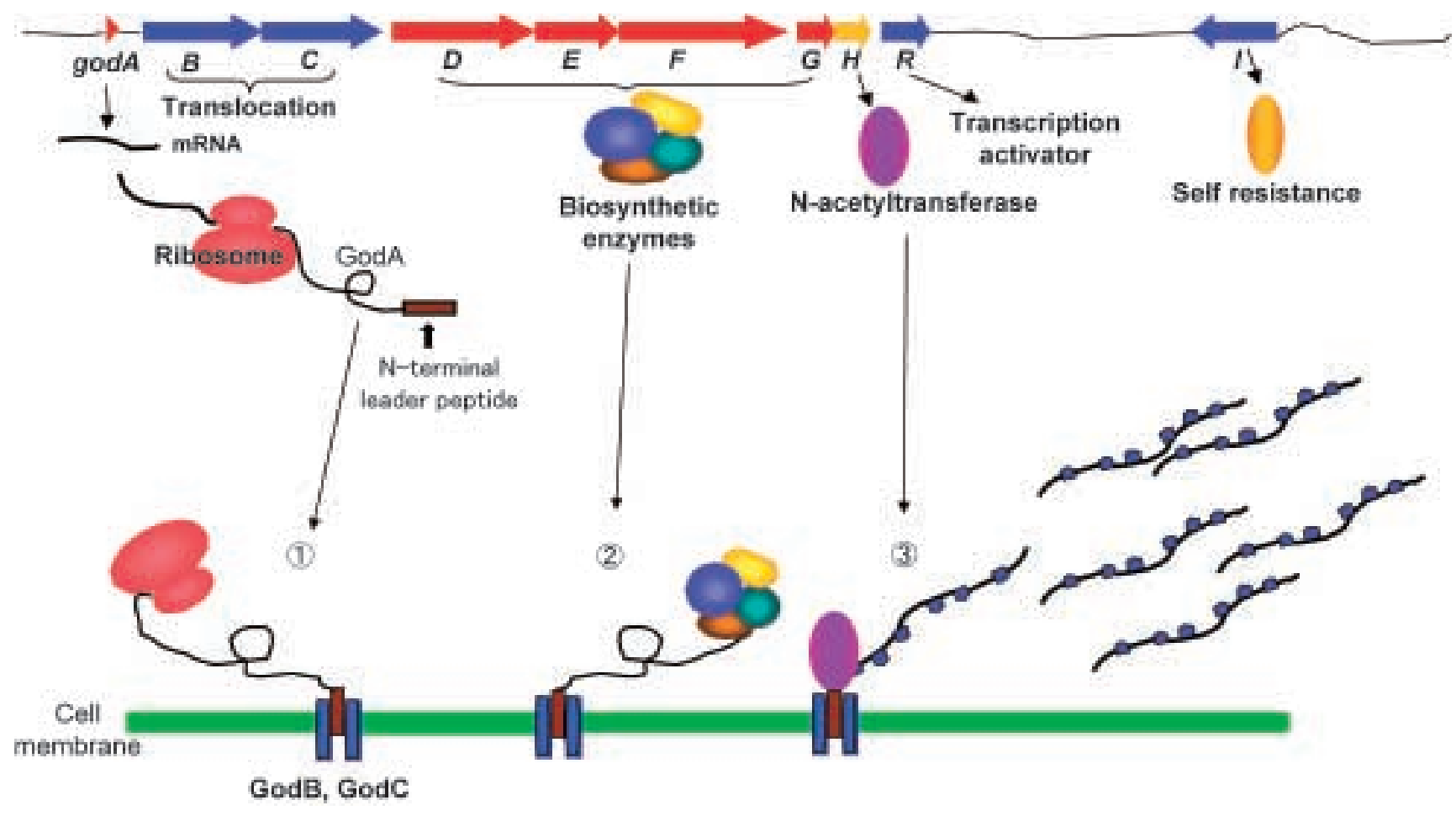

Fig. 8. Biosynthesis of goadsporin. Gene organization of the goadsporin biosynthetic gene cluster in Streptomyces sp. TP-A0584. (1) GodA is translated by ribosomes, and GodB and GodC deliver GodA to the cell membrane. (2) GodD, GodE, GodF, and GodG catalyze the post-translational modification of GodA (hetero cyclization and dehydration). (3) GodB, GodC, or some peptidase digests the leader peptide of GodA, and the N-terminus of goadsporin is acetylated by GodH.

dehydroalanines to generate progoadsporin. Progoadsporin proteolysis is then catalyzed by a peptidase, namely, GodB or GodC. Finally, GodH catalyses N-acetylation of the Nterminus to produce goadsporin. (iii) Heterologous production of goadsporin in S. lividans $^{24)}$

The entire set of goadsporin biosynthetic gene cluster was heterologously expressed in S. lividans by using pGSBC1, and the production of goadsporin in the surrogate host was 
observed. Unexpectedly, the maximum productivity of goadsporin in the heterologous host was more than that in the original hosts. The amount of goadsporin in the heterologous hosts was $347.7 \mathrm{mg} / \mathrm{liter}$ (11 days), whereas that in the original hosts was $126.0 \mathrm{mg} /$ liter (7 days).

(iv) Goadsporin immunity in the producing $\operatorname{strain}^{24)}$

The godI gene is responsible for goadsporin immunity. $S$. lividans harboring godI is resistant to goadsporin, and the godI-disrupted mutant of TP-A0584 is sensitive to goadsporin (Fig. 9A). The godI gene shows sequence similarity to ffh, the signal recognition particle (SRP) in E. coli (44.6\% identity; $74.9 \%$ similarity). SRP is a ribosomal protein that catalyses the targeting of nascent secretory and membrane proteins to the protein translocation apparatus of the cell. SRP homologs have been identical in all living cells. The ffh homologs in S. avermitilis and S. coelicolor A3(2) are also highly conserved with godI $(76.6 \%$ and $75.9 \%$ identity, respectively). The SRP recognizes the signal peptide of secretory or membrane proteins and promotes their delivery to the cytoplasmic membrane. Generally, in bacteria, the SRP forms a complex with ffh and 4.5S RNA. Interestingly, a sequence that shows $91.6 \%$ identity to $4.5 \mathrm{~S}$ RNA in S. lividans is present $235 \mathrm{bp}$ upstream from godI in the genome of the TP-A0584 strain (Fig. 8). Although the exact role of godI remains unknown, our speculative mechanism for goadsporin immunity is shown in Fig. 9B. Goadsporin binds to Ffh to inhibit protein translocation. In the Streptomyces strains, which are sensitive to goadsporin, Ffh cannot induce growth inhibition, secondary metabolism, and/or sporulation in the presence of goadsporin. On the other hand, TP-A0584 contains two types of Ffhs, namely, Ffh and GodI. Goadsporin cannot bind to GodI, and TP-A0584 would substitute GodI for Ffh to maintain the SRP system.

(v) An approach to synthesize the natural goadsporin analogs ${ }^{24)}$

The versatility of the goadsporin biosynthesis machinery has been indicated by the production of goadsporin analogs. The amino acid sequence of godA was changed by sitedirected mutagenesis, and four analogs were isolated from the recombinant gene strains. The mutated godA gene was cloned into the pTYM19 integration vector and integrated into the chromosomal DNA of the godA-disrupted mutant. As shown in Fig. 10A, we produced a new series of goadsporin relatives. G10A is an analog in which the $10^{\text {th }}$ glycine is replaced with alanine. In the S15T analog, the oxazole at the $15^{\text {th }}$ position is changed to methyloxazole. In the T5S analog, the methyl group of the $5^{\text {th }}$ methyloxazole is substituted with hydrogen. The analog $20 \mathrm{~K}$ possesses an additional lysine residue at the carboxyl end. In the goadsporin disc assay, derivatives T5S and S15T retained the parent compound activity of inducing pigment production and sporulation in S. lividans, whereas G10A and $20 \mathrm{~K}$ lost this activity (Fig. 10B). Interestingly, G10A retained the antibiotic activity against $S$. scabies, a potato scab pathogen strain (Fig. 10C). We demonstrated that a wide variety of goadsporin analogs could be produced by this method, and it is expected that this goadsporin biosynthetic machinery could be used for the heterocyclization of oligopeptides.
A

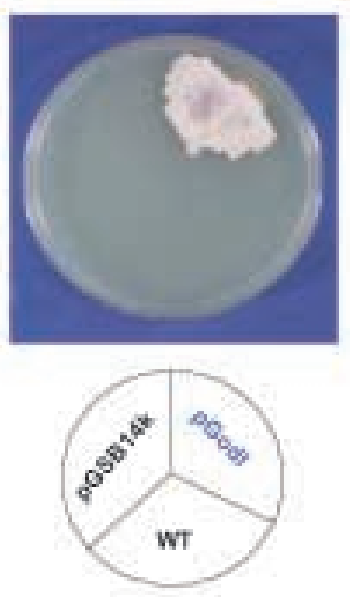

B

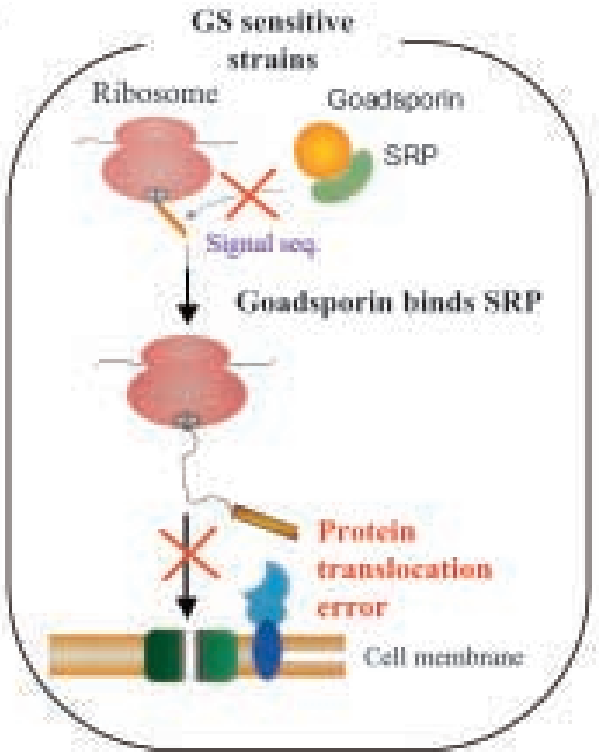

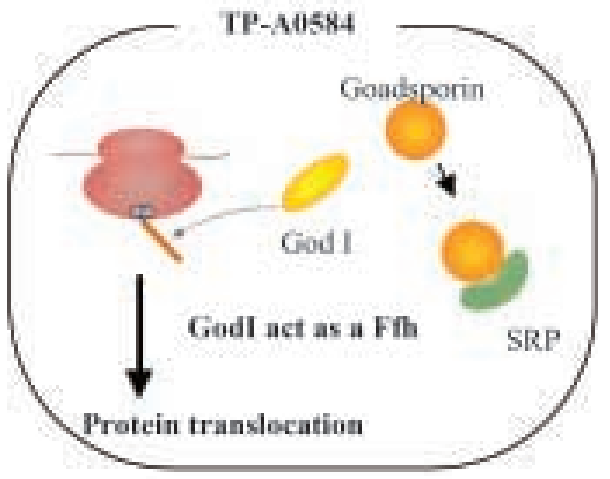

Fig. 9. The self-resistance mechanism mediated by godI in Streptomyces sp. TP-A0584. A: S. lividans harboring pGODI (cloning of godI) grew on a plate containing $30 \mu \mathrm{g}$ goadsporin $\mathrm{ml}^{-1}$, whereas the wild-type strain (S. lividans) and S. lividans harboring pGSB14k (cloning of the entire set of the goadsporin biosynthetic gene cluster, except godI) did not grow. B: The proposed function of GodI and signal recognition particle for goadsporin self-resistance. 
A

\section{Goadsporin}

(t)

Ala-Thr-Val-Ser-Thr-Ile-Leu-Cys-Ser-Gly-Gly-Thr-Leu-Ser-Ser-Ala-Gly-Cys-Val

T5S

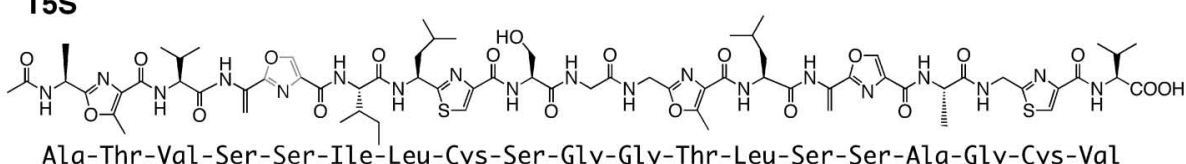

Ala-Thr-Val-Ser-Ser-Ile-Leu-Cys-Ser-Gly-Gly-Thr-Leu-Ser-Ser-Ala-Gly-Cys-Val

G10A

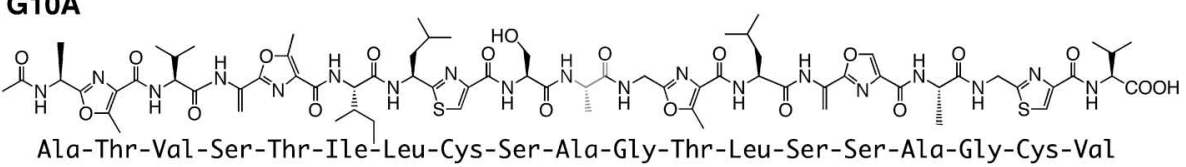

S15T

(1)

Ala-Thr-Val-Ser-Thr-Ile-Leu-Cys-Ser-Gly-Gly-Thr-Leu-Ser-Ser-Ala-Gly-Cys-Val

(2)

Ala-Thr-Val-Ser-Thr-Ile-Leu-Cys-Ser-Gly-Gly-Thr-Leu-Ser-Ser-Ala-Gly-Cys-Val-Lys
B

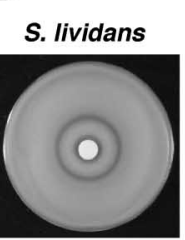

C

S. scabies
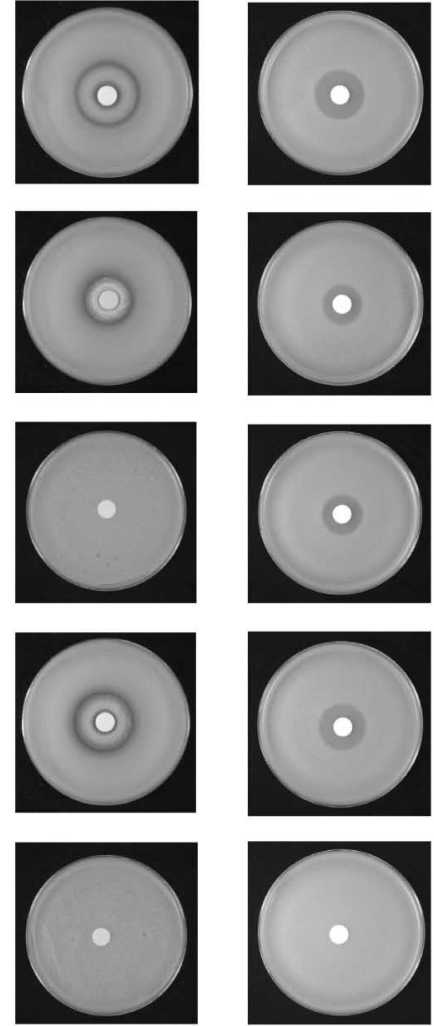

Fig. 10. Chemical structures (A) and biological activity of goadsporin and the selected goadsporin analogs (B, C). GS disc assay with the goadsporin analogs indicates the response against S. lividans TK23 and S. scabies JCM7914. Growth inhibition is indicated by the clear zones of S. lividans TK23 (B) or S. scabies JCM7914 (C).

\section{CONCLUSIONS}

The studies described above summarize our attempts to understand the biosynthesis of two different types of heterocyclic antibiotics-indolocarbazole and goadsporin-on a genetic and biochemical level. A similarity between the two heterocyclic rings is that both are derived from amino acids; nevertheless, the enzymes involved in their biosynthetic pathways are completely different. In indolocarbazole biosynthesis, the pyrrole heterocyclic ring is derived from two molecules of tryptophan, and its formation is catalyzed by $\mathrm{StaD}$ or RebD, a chromopyrrolic acid synthase that was a newly identified hemoprotein in this study. Such a novel synthase-catalyzed heterocyclic ring formation constitutes a unique indolocarbazole biosynthetic pathway. On the other hand, in goadsporin biosynthesis, oxazole and thiazole heterocyclic rings are derived from serine, threonine, or cysteine, and their formation would be catalyzed by GodD, GodE, GodF, and GodG. The amino acid sequences of these enzymes were not significantly similar to those of the known enzymes. These results suggested that goadsporin biosynthesis contains a novel machinery for the formation of a heterocyclic ring, and this biosynthetic pathway is acquired by independent genomic evolution of the TP-A0584 strain. The major secondary metabolites produced by actinomycetes, especially the Streptomyces genus, are polyketide compounds. A number of studies about secondary metabolite biosynthesis in actinomycetes were focused on polyketide compounds. Our biosynthetic pathway has no relevance to the polyketide biosynthetic pathway. I believe that through the characterization of these two distinct biosynthetic pathways, the question - why actinomycetes can produce a wide variety of secondary metabolites?-would be answered convincingly.

\section{ACKNOWLEDGEMENT}

It is my great honor to receive the Hamada Award of the Society for Actinomycetes, Japan (SAJ) in 2005. I would like to thank the past and present members of my laboratory who have carried out a substantial amount of the work described herein. I particularly thank Prof. Tamotsu Furumai and Prof. Yasuhiro Igarashi for their generous support and for providing an opportunity to accomplish my study, and the graduated and present students-Shumpei Asamizu, Shin-ichi Taniguchi, Hirokazu Tabata, Keiko Hayashi, Mizuho 
Nakaho, and Kentaro Haginaka-for working as experimental partners. I would like to acknowledge the continuing encouragement from my former supervisors, namely, Prof. Emeritus Teruhiko Beppu and Prof. Sueharu Horinouchi (The University of Tokyo). I also thank Prof. Haruo Ikeda (Kitasato University), Prof. Ryuji Yoshida, Prof. Tohru Dairi, and Prof. Yasuo Kato (Toyama Prefectural University) for their kind encouragement. Finally, I would like to thank the members of the SAJ for their continuing interest and support.

\section{REFERENCES}

1) Omura, S.; Y. Sasaki, Y. Iwai \& H. Takeshima: Staurosporine, a potentially important gift from a microorganism. J. Antibiot. 48: 535-548, 1995

2) Bush, J. A.; B. H. Long, J. J. Catino, W. T. Bradner, \& K. Tomita: Production and biological activity of rebeccamycin, a novel antitumor agent. J. Antibiot. 40: 668-678, 1987

3) Tamaoki T,; H. Nomoto, I. Takahashi, Y. Kato, M. Morimoto \& Tomita: Staurosporine, a potent inhibitor of phospholipid/Ca ${ }^{++}$dependent protein kinase. Biochem. Biophys. Res. Commun. 135: 397-402, 1986

4) Kase, H.; K. Iwahashi, \& Y. Matsuda: K-252a, a potent inhibitor of protein kinase $\mathrm{C}$ from microbial origin. J. Antibiot. 39: 1059-1065, 1986

5) Kojiri, K.; H. Kondo, T. Yoshinari, H. Arakawa, S. Nakajima, F. Satoh, K. Kawamura, A. Okura, H. Suda, \& M. Okanishi: A new antitumor substance BE-13793C, produced by a streptomycete. Taxonomy, fermentation, isolation, structure determination and biological activity. J. Antibiot. 44: 723-728, 1991

6) Pearce, C. J.; T. W. Doyle, S. Forenza, K. S. Lam, \& D. R. Schroeder: The biosynthetic origins of rebeccamycin. J. Nat. Prod. 51, 937-940, 1988

7) Yang, S. W.; L. J. Lin, G. A. Cordell, P. Wang \& D. G. Corley: $\mathrm{O}-$ and N-methylation in the biosynthesis of staurosporine. J. Nat. Prod. 62: 1551-1553, 1999

8) Lam, K. S.; S. Forenza, T. W. Doyle \& C. J. Pearce: Identification of indolepyruvic acid as an intermediate of rebeccamycin biosynthesis. J. Ind. Microbiol. 6: 291-294, 1990

9) Ohuchi, T.; A. Ikeda-Araki, A. Watanabe-Sakamoto, K. Kojiri, M. Nagashima, M. Okanishi \& H. Suda: Cloning and expression of a gene encoding N-glycosyltransferase (ngt) from Saccharothrix aerocolonigenes ATCC39243. J. Antibiot. 53: 393-403, 2000

10) Onaka, H.; S. Taniguchi, Y. Igarashi \& T. Furumai: Characterization of the biosynthetic gene cluster of rebeccamycin from Lechevalieria aerocolonigenes ATCC 39243. Biosci. Biotechnol. Biochem. 67: 127-138, 2003

11) Onaka, H.; S. Taniguchi, Y. Igarashi \& T. Furumai: Cloning of the staurosporine biosynthetic gene cluster from Streptomyces sp. TP-A0274 and its heterologous expression in
Streptomyces lividans. J. Antibiot. 55: 1063-1071, 2002

12) Sanchez, C.; A. I. Butovich, F. A. Brana, J. Rohr, C. Mendez \& J. A. Salas: The biosynthetic gene cluster for the antitumor rebeccamycin: Characterization and generation of indolocarbazole derivatives. Chem. Biol. 9: 519-531, 2001

13) Hyun C. G,; T. Bililign, J. Liao \& J. S. Thorson: The biosynthesis of indolocarbazoles in a heterologous E. coli host. Chembiochem. 4: 114-7, 2003

14) Sanchez C,; L. Zhu, A. F. Brana, A. P. Salas, J. Rohr, C. Mendez \& J. A. Salas JA: Combinatorial biosynthesis of antitumor indolocarbazole compounds. Proc. Natl. Acad. Sci. USA. 102: 461-466, 2005

15) Onaka, H.; H. Tabata, Y. Igarashi, Y. Sato \& T. Furumai: Goadsporin, a chemical substance which promotes secondary metabolism and morphogenesis in streptomycetes. I. Purification and characterization. J. Antibiot. 54: 1036-1044, 2001

16) Igarashi Y.; Y. Kan, K. Fujii, T. Fujita, K. Harada, H. Naoki, H. Tabata, H. Onaka \& T. Furumai:. Goadsporin, chemical substance which promotes secondary metabolism and morphogenesis in streptomycetes II. structure determination. J. Antibiot. 54: 1045-1053, 2001

17) Onaka, H.; S. Taniguchi, H. Ikeda, Y. Igarashi \& T. Furumai: pTOYAMAcos, pTYM18, and pTYM19, actinomyceteEscherichia coli integrating vectors for heterologous gene expression. J. Antibiot. 56: 950-956, 2003

18) Onaka H.; S. Asamizu, Y. Igarashi, R. Yoshida \& T. Furumai: Cytochrome $\mathrm{P} 450$ homolog is responsible for $\mathrm{C}-\mathrm{N}$ bond formation between aglycone and deoxysugar in the staurosporine biosynthesis of Streptomyces sp. TP-A0274. Biosci. Biotechnol. Biochem. 69: 1753-1759, 2005

19) Howard-Jones A. R. \& C. T. Walsh: Staurosporine and rebeccamycin aglycones are assembled by the oxidative action of StaP, StaC, and RebC on chromopyrrolic acid. J. Am. Chem. Soc. 128:12289-12298, 2006

20) Asamizu, S.; Y. Kato, Y. Igarashi, T. Furumai \& H. Onaka: Direct formation of chromopyrrolic acid from indole-3-pyruvic acid by $\mathrm{StaD}$, a novel hemoprotein in indolocarbazole biosynthesis, Tetrahedron lett. 47: 473-475, 2006

21) Howard-Jones A. R. \& C. T. Walsh: Enzymatic generation of the chromopyrrolic acid scaffold of rebeccamycin by the tandem action of RebO and RebD. Biochemistry. 44: 1565215666, 2005

22) Nishizawa T, S. Gruschow, D. H. Jayamaha, C. NishizawaHarada \& D. H. Sherman: Enzymatic assembly of the bisindole core of rebeccamycin. J. Am. Chem. Soc. 128: 724-725, 2006

23) Onaka H.; Characterization of the biosynthetic pathway of heterocyclic antibiotics and production of unnatural natural compounds., Nagase science and technology foundation report 2004, 16: 77-86, 2005 ( in Japanese with English abstract)

24) Onaka, H.; M. Nakaho, K. Hayashi, Y. Igarashi \& T. Furumai: Cloning and characterization of goadsporin biosynthetic gene cluster from Streptomyces sp. TP-A0584. Microbiology, 151: 3923-3933, 2005 